\title{
Probing the Nature of Short Swift Bursts via Deep INTEGRAL Monitoring of GRB 050925
}

\author{
T. Sakamoto ${ }^{\mathrm{a}, \mathrm{b}}$, L. Barbier ${ }^{\mathrm{c}}$, S. D. Barthelmy ${ }^{\mathrm{c}}$, J. R. Cummings ${ }^{\mathrm{a}, \mathrm{b}}$, E. E. \\ Fenimore $^{\mathrm{d}}$, N. Gehrels ${ }^{\mathrm{c}}$, H. A. Krimm ${ }^{\mathrm{a}, \mathrm{e}}$, C. B. Markwardt ${ }^{\mathrm{a}, \mathrm{f}}$, D. M. \\ Palmer $^{\text {d }}$, A. M. Parsons ${ }^{\text {c }}$, G. Sato ${ }^{g}$, M. Stamatikos ${ }^{\text {h}}$, J. Tueller ${ }^{\mathrm{c}}$ \\ ${ }^{a}$ CRESST and NASA Goddard Space Flight Center, Greenbelt, MD 20771 \\ ${ }^{b}$ Joint Center for Astrophysics, University of Maryand, Baltimore County, Baltimore, \\ MD 21250 \\ ${ }^{c}$ NASA Goddard Space Flight Center, Greenbelt, MD 20771 \\ ${ }^{d}$ Los Alamos National Laboratory, P.O. Box 1663, Los Alamos, NM, 87545 \\ ${ }^{e}$ Universities Space Research Association, Columbia, MD 21044 \\ ${ }^{f}$ Department of Astronomy, University of Maryland, College Park, MD 20742 \\ ${ }^{g}$ Institute of Space and Astronautical Science, JAXA, Kanagawa 229-8510, Japan \\ ${ }^{h}$ Oak Ridge Associated Universities, P.O. Box 117, Oak Ridge, Tennessee 37831
}

\begin{abstract}
We present results from Swift, XMM-Newton, and deep INTEGRAL monitoring in the region of GRB 050925. This short Swift burst is a candidate for a newly discovered soft gamma-ray repeater (SGR) with the following observational burst properties: 1$)$ galactic plane $\left(\mathrm{b}=-0.1^{\circ}\right)$ localization, 2) 150 msec duration, and 3) a blackbody rather than a simple power-law spectral shape (with a significance level of $97 \%$ ). We found two possible X-ray counterparts of GRB 050925 by comparing the X-ray images from Swift XRT and XMM-Newton. Both X-ray sources show the transient behavior with a power-law decay index shallower than -1 . We found no hard X-ray emission nor any additional burst from the location of GRB 050925 in $\sim 5 \mathrm{Ms}$ of INTEGRAL data. We discuss about the three BATSE short bursts which might be associated with GRB 050925, based on their location and the duration. Assuming GRB 050925 is associated with the $\mathrm{H}_{I I}$ regions (W 58) at the galactic longitude of $l=70^{\circ}$, we also discuss the source frame properties of GRB 050925.
\end{abstract}

Keywords: gamma ray, burst 


\section{Introduction}

The origin of the short ( $<2$ seconds) class of gamma-ray bursts (GRBs) is receiving huge attention in the field of high-energy astrophysics. Thanks to the rapid position notices and response by HETE-2 and Swift, afterglow emission has been found for a handful of short GRBs (e.g., Berger et al., 2007). Less than arc-second positions, which are provided by X-ray and optical afterglows, enable us to study the host galaxies of short GRBs. Surprisingly, unlike the long duration GRBs which always have host galaxies with a high star forming rate (Bloom et al., 2002), short GRBs emerge from both star-forming and non-star forming galaxies (e.g., Villasenor et al. , 2005). This suggests that a substantial range of lifetimes is needed for the progenitors of short GRBs. This discovery tightens the case for a different origin for short and long GRBs.

On the other hand, some fraction of short bursts might be from local and extra galactic flares of soft gamma-ray repeaters (SGRs). SGRs are believed to be highly magnetized isolated neutron stars (Duncan \& Thompson, 1992). They produce short spikes (a few tens of milliseconds) (Woods, 2003) and sometimes a giant flare (Hurley et al. , 1999; Palmer et al. , 2005) in $\gamma$ rays. Although it is possible to detect giant flares of extragalatic SGRs from nearby galaxies, their fraction of such flares among short GRBs is still not clear (e.g., Palmer et al. , 2005; Lazzati et al. , 2005), Furthermore, a small flare from a previously unknown SGR in the galaxy might be detected as a short burst in $\gamma$-rays.

\section{GRB 050925}

\subsection{Swift/BAT Prompt Emission}

On 25 September 2005, the Swift Bust Alert Telescope (BAT) instrument detected GRB 050925, which only lasted for $\sim 100 \mathrm{~ms}$ and possibly consists of two pulses (Figure 1). The BAT ground analysis position of this burst is (R.A., Dec. $)=\left(20^{\mathrm{h}} 13^{\mathrm{m}} 56.9^{\mathrm{s}}, 34^{\circ} 19^{\prime} 48^{\prime \prime}\right)(\mathrm{J} 2000)$ which corresponds to the galactic coordinate of $(l, b)=\left(72.320^{\circ},-0.101^{\circ}\right)$ (Sakamoto et al. , 2010). Its location is in the galactic plane $\left(b=-0.1^{\circ}\right)$. The $T_{90}$ and $T_{50}$ duration is $90 \mathrm{~ms}$ and $40 \mathrm{~ms}$ respectively. The fluence in the $104 \mathrm{~ms}$ time window $\left(T_{100}\right.$ interval) in the $15-150 \mathrm{keV}$ band is $(7.7 \pm 0.9) \times 10^{-8} \mathrm{ergs}^{-2}$. The peak flux in the $10 \mathrm{~ms}$ time window is estimated to be $(1.2 \pm 0.1) \times 10^{-6} \mathrm{ergs} \mathrm{cm}^{-2}$ $\mathrm{s}^{-1}$. 
As seen in Figure 2, the prompt emission spectrum of this burst shows a better fit to a blackbody (BB) spectrum with a temperature of $15.1 \pm 0.5$ $\mathrm{keV}\left(\chi^{2} /\right.$ dof $\left.=75.3 / 57\right)$ over a simple power-law $(\mathrm{PL})$ spectrum $\left(\chi^{2} / \mathrm{dof}=\right.$ $87.7 / 57)$. To quantify the significance of this improvement, we performed 10,000 spectral simulations assuming the best-fit spectral parameters in a simple power-law model and determined in how many cases the blackbody fit gives $\chi^{2}$ improvements of equal or greater than $\Delta \chi^{2}=\chi^{2}(\mathrm{PL})-\chi^{2}(\mathrm{BB})$ $=12.4$ over the simple power-law model. We found equal or higher improvements in $\chi^{2}$ in 691 simulated spectral out of 10,000. Thus, the chance probability of having an equal or higher $\Delta \chi^{2}$ of 12.4 with the blackbody model when the parent distribution is a simple power-law model is $7 \%$. A blackbody with a temperature of $\sim 10 \mathrm{keV}$ is a typical spectral model for short SGR bursts in the BAT energy range (e.g., Olive et al. , 2005; Fenimore et al. , 1994). GRB 050925 belongs to a softer region compared to the other short GRBs detected by BAT in the hardness-duration plot (Figure 3).

The spectral lag using the $10 \mathrm{~ms}$ light curves between the $50-100 \mathrm{keV}$ and the $15-50 \mathrm{keV}$ band is $0.012 \pm 0.055 \mathrm{~s}(1 \sigma)$ which is consistent with zero. This negligible spectral lag of GRB 050925 is consistent with either short GRBs

(e.g. Norris et al. , 2010) or short bursts from SGRs (e.g., Nakagawa et al. , 2007).

\subsection{X-ray Observations}

The Swift X-ray Telescope (XRT) started observing the BAT error circle about 100 seconds after the burst with a net exposure of $30 \mathrm{ksec}$. XRT found four sources (labeled as Src \#1 to \#4 as shown in left panel of Figure 4) near and inside the BAT 90\% error circle. Only Src \#4 is located completely inside the BAT error circle. The positions of Src \#2 and Src \#3 are coincident with stars in the guide star catalog (GSC) version 2.2 (N033223385350 and N033223389), and are also detected by Swift UV-optical telescope (right panel of Figure 41). Therefore, these two X-ray sources are stars, and not associated with GRB 050925.

The XMM-Newton observed the field of GRB 050925, starting from 17 days after the BAT trigger, for $26.6 \mathrm{ksec}$ (Rea et al., 2005). The XMM EPIC mosaic image (middle panel of Figure 4) shows detections of Src \#2, Src \#3 and Src \#4. However, there is no detection of Src \#1 in the XMM image. Based on the count-rate in the sensitivity map at the location of Src $\# 1$, the upper-limit of the flux is $1 \times 10^{-14} \mathrm{erg} \mathrm{cm}^{-2} \mathrm{~s}^{-1}(0.2-12 \mathrm{keV})$. Using 


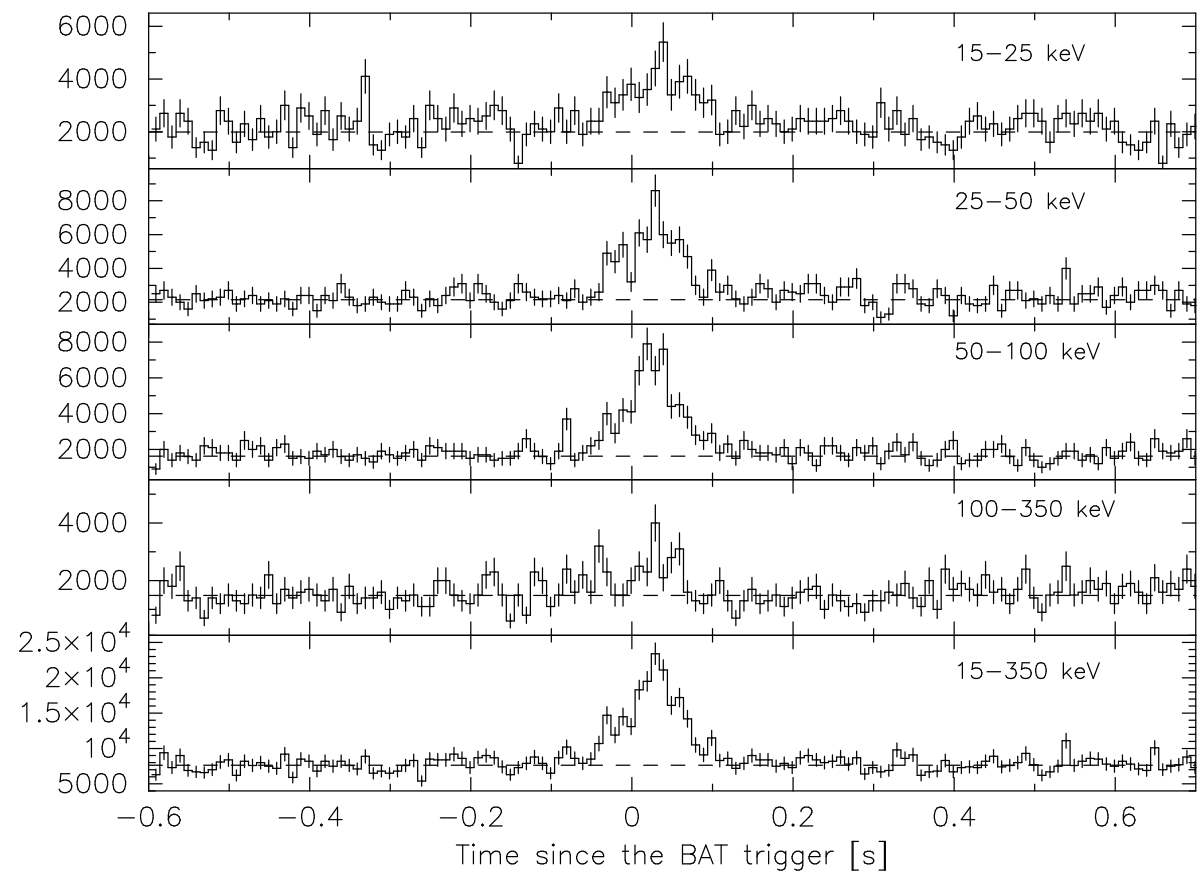

Figure 1: The BAT five channel light curves in 10 msec binning. The background has not been subtracted.

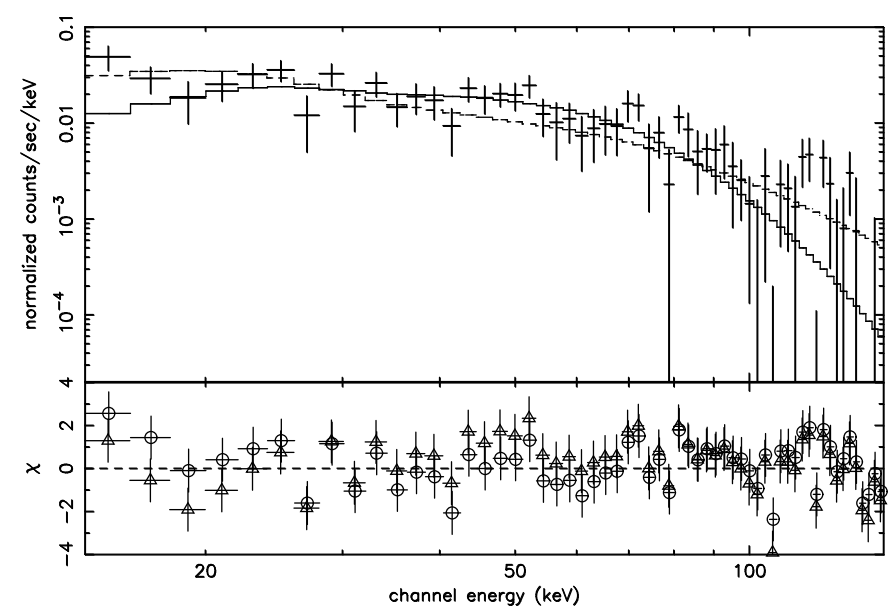

Figure 2: The BAT time-integrated spectrum of the burst (104 msec duration). The best fit with a blackbody model is shown in a solid line (upper) with circles in a residual panel (bottom), whereas, the best fit with a simple power-law model is shown in a dashed line (upper) with triangles in a residual panel (bottom). 


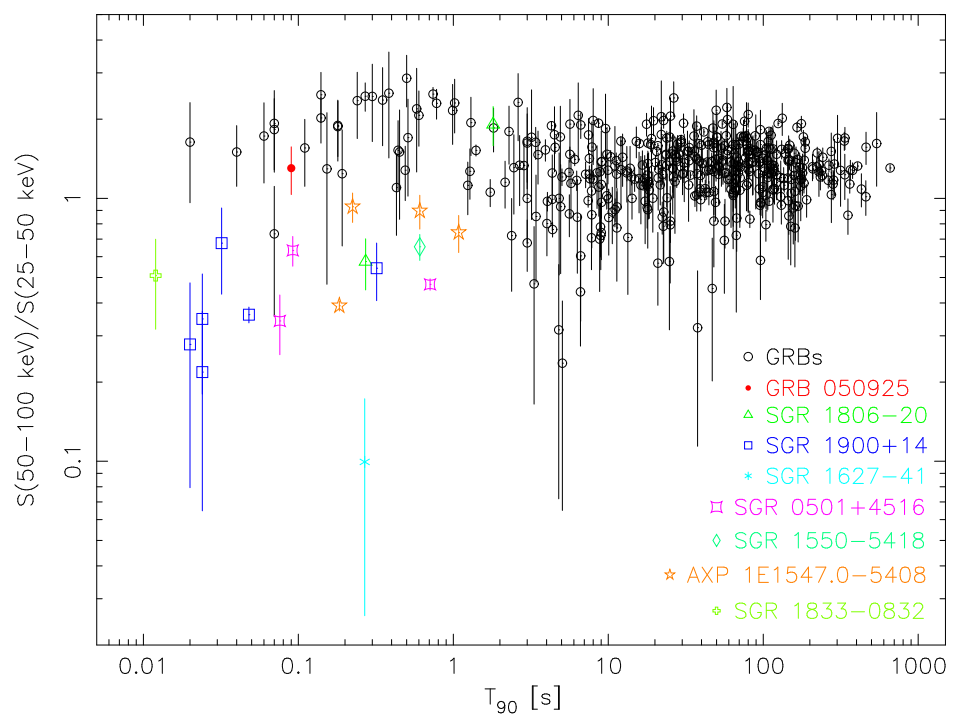

Figure 3: The BAT fluence ratio between the $50-100 \mathrm{keV}$ and $25-50 \mathrm{keV}$ band vs. $T_{90}$ duration. The BAT GRBs (black open circles), GRB 050925 (red filled circle), and short bursts from SGRs/AXPs, which are detected by BAT, are shown in different marks and colors.

the WebPIMMS1 1 and assuming the same absorbed power-law spectrum in the flux calculation of the XMM count-rate $\left(N_{H}=3 \times 10^{20}\right.$ and a power-law photon index of 1.7), the XRT count-rate of $(2.3 \pm 0.3) \times 10^{-3} \mathrm{c} / \mathrm{s}$ corresponds to $(1.05 \pm 0.14) \times 10^{-13} \mathrm{erg} \mathrm{cm}^{-2} \mathrm{~s}^{-1}$. By combining the XRT detection and the corresponding upper-limit in the XMM observation of Src \#1, we found that the source has to decline steeper than a power-law index of -0.7 . Although Src \#1 is just outside the BAT error circle, its transient nature makes it a possible X-ray counterpart of GRB 050925.

The XRT count-rate of Src \#4, which is the only X-ray source inside the BAT error circle, is $(7.0 \pm 1.7) \times 10^{-4} \mathrm{c} / \mathrm{s}$. The estimated absorbed flux using the same spectral parameters of Src \#1 is $(3.2 \pm 0.8) \times 10^{-14} \mathrm{erg} \mathrm{cm}^{-2} \mathrm{~s}^{-1}$. On the other hand, the estimated flux in the XMM EPIC mosaic image is $(4.2 \pm 2.4) \times 10^{-15} \mathrm{erg} \mathrm{cm}^{-2} \mathrm{~s}^{-1}$. Therefore, the source shows a decline in the flux by a power-law index of -0.6 between these two observations.

Based on analysis of these observations from XRT and XMM, both Sre

\footnotetext{
${ }^{1}$ http://heasarc.gsfc.nasa.gov/Tools/w3pimms.html
} 

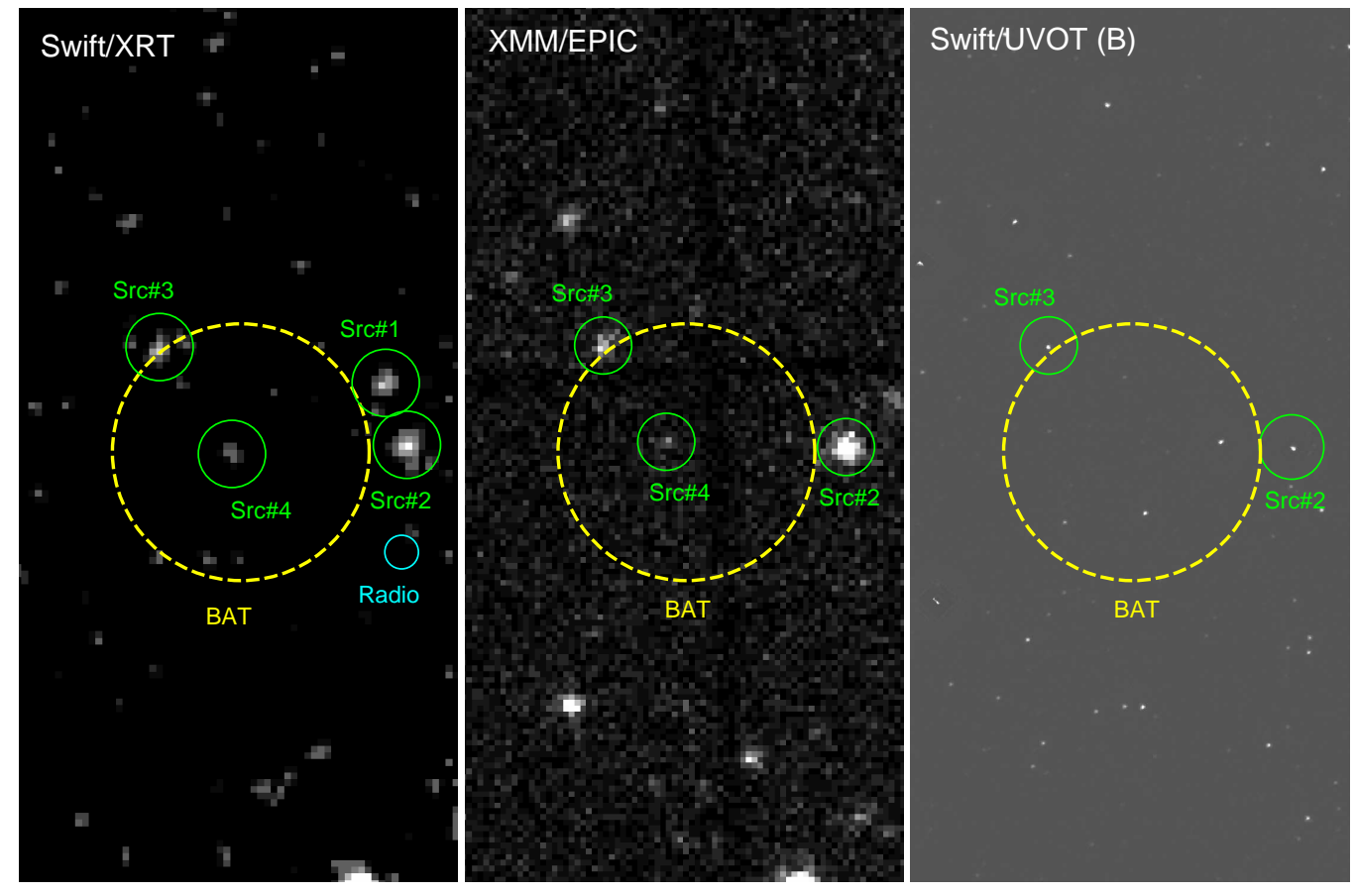

Figure 4: The X-ray and B band images of the field of GRB 050925 (left: Swift XRT, middle: XMM-Newton, and right: Swift UVOT B filter). The larger dashed yellow circle is the BAT $90 \%$ error circle based on the ground analysis. The location of a radio source, which mentioned in section 2.3 , is shown in a cyan circle.

\#1 and Src \#4 show a decline in the flux between these two observations. Therefore, at this stage, it is not possible to identify which source is the X-ray counterpart of GRB 050925.

\subsection{Optical, Infrared and Radio Observations}

The 2-m Faulkes North Telescope followed up GRB 050925 at 3.3 minutes after the BAT trigger time. No new optical source found inside the BAT flight location with the upper limit of $19 \mathrm{mag}$ in $\mathrm{R}$ (Guidorzi et al. , 2005). The PAIRITEL $1.3-\mathrm{m}$ telescope observed GRB 050925 at 17.6 hours after the trigger in infrared bands. No new source was found within $1^{\prime \prime}$ of the radio source (see below about the radio source) with $5 \sigma$ upper limits of $18.3 \mathrm{mag}$, $18.5 \mathrm{mag}$ and $19.3 \mathrm{mag}$ in $\mathrm{K}_{\mathrm{S}}$, J and $\mathrm{H}$, respectively (Bloom, 2005). Swift UVOT also found no new source within the BAT error circle with the $3 \sigma$ 
upper limits of $20.2 \mathrm{mag}, 21.8 \mathrm{mag}$ and $21.2 \mathrm{mag}$ in $\mathrm{V}, \mathrm{B}$ and $\mathrm{U}$, respectively (Rosen et al. , 2005).

The Westerbork Synthesis Radio Telescope observed GRB 050925 in 4.216.3 hours after the BAT trigger and found no radio source within the BAT error circle with a $3 \sigma$ upper limit of $72 \mu \mathrm{Jy}$. However, they note a bright radio source, which turns out to be a constant source based on 2 nd observation $(\sim 6$ days after the trigger), just outside the BAT error circle at (R.A., Dec.) = $\left(20^{\mathrm{h}} 13^{\mathrm{m}} 47.8^{\mathrm{s}}, 34^{\circ} 18^{\prime} 38^{\prime \prime}\right)$ (van der Horst et al. , 2005a, b). The location of this radio source does not match with two possible X-ray sources associated with GRB 050925 and discussed in section 2.2.

\subsection{Search for Persistent Hard X-ray Emission by INTEGRAL}

The INTEGRAL data of the IBIS/ISGRI have been analyzed using the INTEGRAL Off-line Scientific Analysis package (OSA v7.0). The data from December 2002 to May 2008, when the location of GRB 050925 is in the field of view of the IBIS, have been processed to cover the pre and the post burst periods of GRB 050925. The ISGRI mosaic images are created for the pre-burst period (from December 21, 2002 to July 2, 2005), the post-burst period (from November 10, 2005 to May 23, 2008) and the total period (from December 21, 2002 to May 23, 2008) in the 20-40 keV, 40-60 keV, 60-100 keV, 100-200 keV, 20-60 keV and 20-200 keV bands. The total exposure of the mosaic images are $2.4 \mathrm{Ms}, 2.5 \mathrm{Ms}$ and $4.9 \mathrm{Ms}$ for the pre-burst, the post-burst and the total period respectively. The intensity maps and the significance maps of six energy bands and different periods are shown in Figure 5. The significance of the location of GRB 050925 is $<1.3 \sigma$ in all mosaic images. Therefore, no persistent hard X-ray emission has found in the ISGRI data.

Using the ISGRI sensitivity estimate of (Krivonos et al. , 2010), $f_{\text {lim }}^{5 \sigma}=$ $0.77 \times(T / M s)^{-0.5} \mathrm{mCrab}$, we estimated the $5 \sigma$ upper limits of the persistent hard X-ray emission at the location of GRB 050925 in the pre-burst, the post-burst and the total period as $0.50 \mathrm{mCrab}, 0.49 \mathrm{mCrab}$ and $0.35 \mathrm{mCrab}$ respectively.

\subsection{Search for short bursts by INTEGRAL}

To search for additional bursts from GRB 050925, we create $100 \mathrm{~ms}$ light curves in the 20-40 keV, 40-60 keV, 60-100 keV, 100-200 keV, 20-60 keV and 20-200 keV bands using ii_light task of the OSA software package. We set the criteria for a possible burst to have $>7 \sigma$ in a light curve bin in the 20-40 keV and the 40-60 keV bands simultaneously to avoid spurious short 
events which we usually only see in a single band 2 . We found no possible short burst at the location of GRB 050925 in our 4.9 Ms data.

\section{Discussion}

We search the BATSE Gamma-Ray Burst Catalog 3 for additional bursts from the location of GRB 050925. We found seven events which their error radii match the location of GRB 050925. Furthermore, we found three candidates (out of seven) which have the BATSE $T_{90}$ shorter than $2: 4$ (Table 1). Figure 6, 8 shows the BATSE light curves of those events. From the duration point of view, the BATSE trigger 878 (4B 911007) has the shortest duration among the candidates. However, its duration is still three times longer than that of GRB 050925. Assuming these three triggers are associated with GRB 050925, the burst rate is estimated to be $0.33 \mathrm{yr}^{-1}$ (the BATSE catalog contains the events detected in 9 years of its operation). Therefore, there is no surprise that we do not find any additional burst in the 4.9 Ms INTEGRAL data.

Around the galactic longitude of $l=70^{\circ}$, there is a complex of $\mathrm{H}_{\mathrm{II}}$ regions (W 58) at a distance of $8.8 \mathrm{kpc}$ (Georgelin \& Georgelin, 1976). Assuming GRB 050925 is associated with the same $\mathrm{H}_{\mathrm{II}}$ regions and therefore at a distance of $8.8 \mathrm{kpc}$, we discuss the source frame properties of the burst and its possible X-ray counterparts. The total radiated energy is $7 \times 10^{38} \mathrm{ergs}$ and the peak luminosity is $1 \times 10^{40} \mathrm{erg} \mathrm{s}^{-1}$ for the burst. Therefore, the radiated energy and the peak luminosity are similar to those of weak short bursts from known SGRs. Assuming the same distance, the X-ray luminosities of Src \#1 and Src \#4 during the XRT observation are $9 \times 10^{32} \mathrm{erg} \mathrm{s}^{-1}$ and $3 \times 10^{32}$ $\mathrm{erg} \mathrm{s}^{-1}$ respectively. Since the X-ray afterglow luminosities of the SGR short bursts are $10^{34}-10^{35} \mathrm{erg} \mathrm{s}^{-1}$ (Nakagawa et al. , 2008), the X-ray luminosity of Src \#1 could be more consistent compared to that of Src \#4. However, note that the X-ray luminosity of Src \#1 is still order of magnitude lower than the typical X-ray afterglow luminosities of the SGR short bursts. We also want to note that the shallow decay index $(<-1)$ which we found for

\footnotetext{
${ }^{2}$ Since a short burst from a SGR has a spectrum from a few keV up to few $100 \mathrm{keV}$, we should see the event at light curves in a several continuous energy bands.

${ }^{3}$ http://www.batse.msfc.nasa.gov/batse/grb/catalog/current/

${ }^{4}$ We exclude one event, trigger ID 2468, because its duration information is not available in the catalog
} 
Table 1: Three candidates found in the BATSE catalog.

\begin{tabular}{ccccccc}
\hline GRB name & ID & $\begin{array}{c}\text { Trigger time } \\
(\mathrm{UT})\end{array}$ & $\begin{array}{c}\text { R.A. } \\
(\mathrm{deg})\end{array}$ & $\begin{array}{c}\text { Dec. } \\
(\mathrm{deg})\end{array}$ & $\begin{array}{c}\text { Error radius } \\
(\mathrm{deg})\end{array}$ & $\begin{array}{c}T_{90} \\
(\mathrm{~s})\end{array}$ \\
\hline 4B 911007 & 878 & $15: 32: 09.2$ & 308.91 & 40.59 & 19.67 & 0.384 \\
4B 920722 & 1719 & $21: 21: 27.2$ & 321.19 & 36.75 & 15.71 & 1.047 \\
4B 930219E & 2205 & $20: 54: 06.0$ & 302.41 & 20.02 & 15.98 & 1.152 \\
\hline
\end{tabular}

both Src \#1 and Src \#4 is common for the X-ray afterglow of SGR short bursts (Nakagawa et al. , 2008).

Assuming the distance of $8.8 \mathrm{kpc}$, the upper limit of the hard X-ray luminosity will be $<3 \times 10^{34} \mathrm{erg} \mathrm{s}^{-1}$. According to the detections of the hard X-ray emission of five magnetars by INTEGRAL (Götz et al. , 2006), the hard X-ray luminosities are in the range of $5 \times 10^{34}-4 \times 10^{35} \mathrm{erg} \mathrm{s}^{-1}$. Therefore, our upper limit at the location of GRB 050925 might not deep enough to conclude a non-existence of the hard X-ray emission. We probably need to revisit the INTEGRAL data in the future with more data or to wait for the data from the future focusing hard X-ray mission NuST AR to answer this question.

We would like to thank the anonymous reviewers for comments and suggestions that materially improved the paper.

\section{References}

A New Population of High-Redshift Short-Duration Gamma-Ray Bursts, Berger, E., Fox, D. B., Price, P. A., et al. 2007, ApJ, 664, 1000-1010

The Observed Offset Distribution of Gamma-Ray Bursts from Their Host Galaxies: A Robust Clue to the Nature of the Progenitors, Bloom, J. S., Kulkarni, S. R., Djorgovski, S. G. 2002, AJ, 123, 1111-1148

GRB/SGR 050925: IR Observations, Bloom, J. 2005, GCN Circ. 4042

Formation of very strongly magnetized neutron stars - Implications for gamma-ray bursts, Duncan, R. C., Thompson, C. 1992, ApJL, 392, 9-13

The X-ray spectrum of the soft gamma repeater 1806-20, Fenimore, E. E., Laros, J. G., Ulmer, A. 1994, ApJ, 432, 742-752 
The spiral structure of our Galaxy determined from $H$ II regions, Georgelin, Y. M., Georgelin, Y. P. 1976, A\&A, 49, 57-79

Magnetars as persistent hard X-ray sources: INTEGRAL discovery of a hard tail in SGR 1900+14, Götz, D., Mereghetti, S., Tiengo, A., Esposito, P. 2006, A\&A, 449, L31-L34

GRB050925: Faulkes Telescope North R-band limit, Guidorzi, C., Mundell, C. G., Gomboc, A. et al. 2005, GCN Circ. 4035

A giant periodic flare from the soft $\gamma$-ray repeater SGR1900+14, Hurley, K., Cline, T., Mazets, E. et al. 1999, Nature, 397, 41-43

INTEGRAL/IBIS 7-year All-Sky Hard X-Ray Survey. Part I: Image Reconstruction, Krivonos, R., Revnivtsev, M., Tsygankov, S. et al. 2010, A\&A in press (arXiv:1006.2463)

Soft gamma-ray repeater giant flares in the BATSE short gamma-ray burst catalogue: constraints from spectroscopy, Lazzati, D., Ghirlanda, G., Ghisellini, G. 2005, MNRAS, 362, L8-L12

A Comprehensive Study of Short Bursts from SGR1806-20 and SGR1900+14 Detected by HETE-2, Nakagawa, Y. E., Yoshida, A., Hurley, K. et al. 2007, PASJ, 59, 653-678

The Swift Discovery of X-Ray Afterglows Accompanying Short Bursts from SGR 1900+14, Nakagawa, Y. E., Sakamoto, T., Sato, G. et al. 2008, ApJ, 681, L89-L92

Threshold for Extended Emission in Short Gamma-ray Bursts, Norris, J. P., Gehrels, N., Scargle, J. D. 2010, ApJ, 717, 411-419

Time-resolved X-Ray Spectral Modeling of an Intermediate Burst from SGR 1900+14 Observed by HETE-2 FREGATE and WXM, Olive, J.-F., Hurley, K. Sakamoto, T. et al. 2005, ApJ, 616, 1148-1158

A giant $\gamma$-ray flare from the magnetar SGR 1806 - 20, Palmer, D. M., Barthelmy, S., Gehrels, N. et al. 2005, Nature, 434, 1107-1109

GRB/SGR 050925: XMM-Newton ToO observation, Rea, N., Costantini, E., Israel, G. 2005, GCN Circ. 4264 
GRB050925: Swift UVOT upper limits, Rosen, S., Holland, S. T., Marshall, F. et al. 2005, GCN Circ. 4038

The Second Swift BAT Gamma-Ray Burst Catalog, Sakamoto, T., Barthelmy, S. D., Baumgartner, W. H. et al. 2010, submitted to ApJS

GRB 050925: WSRT Radio Observations, van der Horst, A. J. 2005a, GCN Circ. 4039

GRB 050925: Second Epoch WSRT Radio Observations, van der Horst, A. J. 2005b, GCN Circ. 4057

Discovery of the short -ray burst GRB 050709, Villasenor, V., Lamb, D. Q., Ricker, G. R. et al. 2005, Nature, 437, 855-858

The Effects of Burst Activity on Soft Gamma Repeater Pulse Properties and Persistent Emission, Woods, P. M. 2003, AIP Conf. Proc., 662, 561-569 

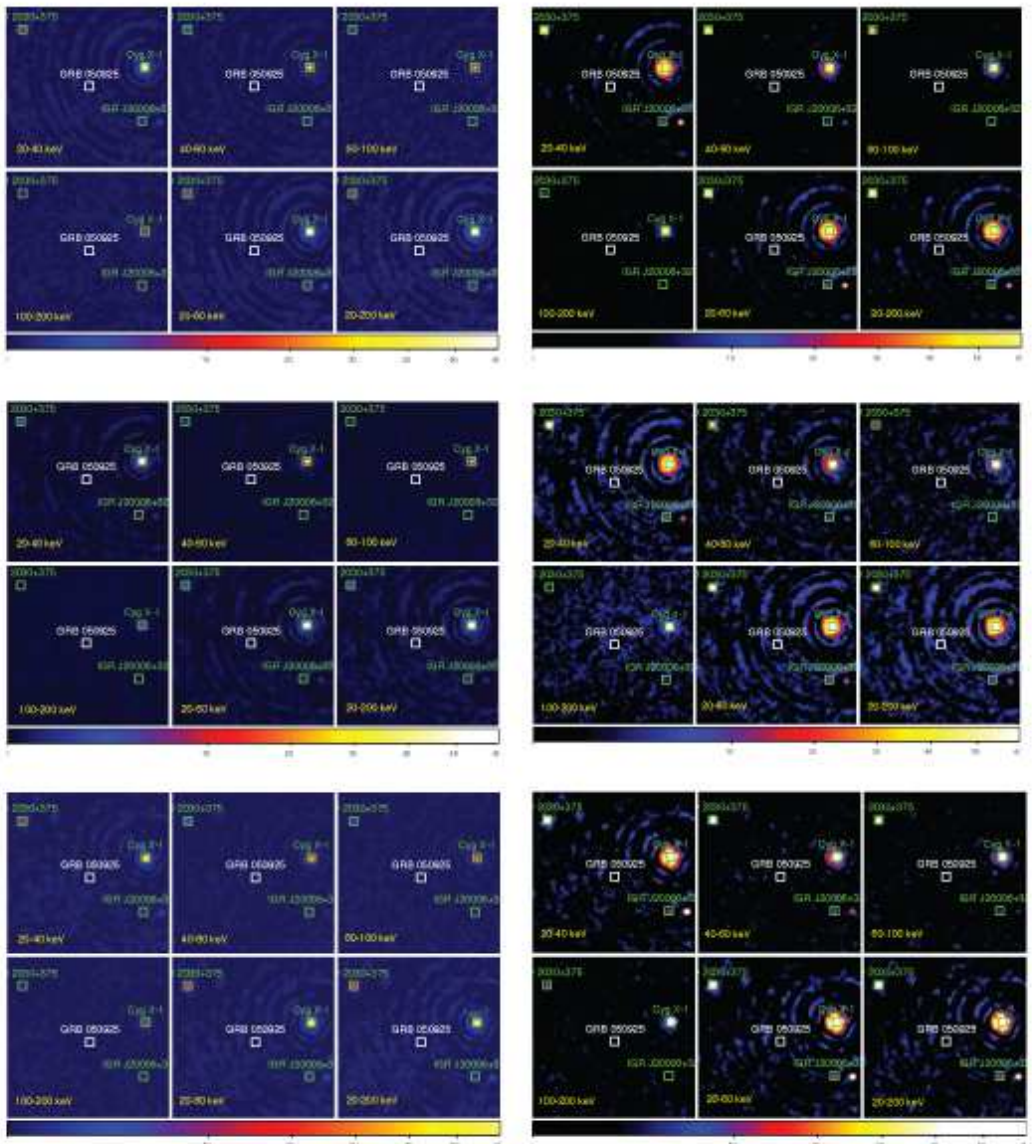

Figure 5: The left column figures show the INTEGRAL ISGRI intensity maps of the total (top), the pre-burst (middle), and the post-burst (bottom) period. The right column figures show the INTEGRAL ISGRI significance maps of the total (top), the pre-burst (middle) and the post-burst (bottom) period. Each of the panels of the figure correspond to the different energy bands: the top panel from left to right: $20-40 \mathrm{keV}, 40-60 \mathrm{keV}$ and 60-100 keV band, and the bottom panel from left to right: 100-200 keV, 20-60 keV and 20-200 keV band. The location of GRB 050925 is shown in a white square. 


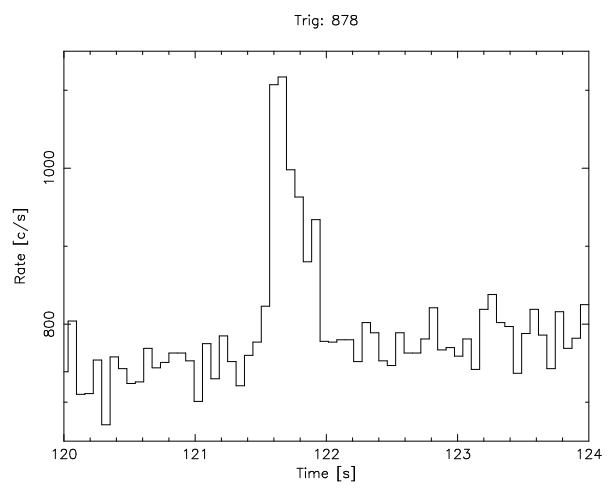

Figure 6: $64 \mathrm{~ms}$ light curve of the BATSE trigger 878 .

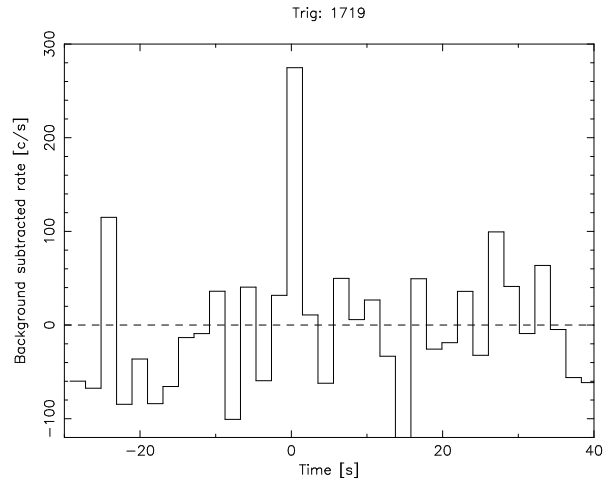

Figure 7: $1 \mathrm{~s}$ light curve of the BATSE triger 1719.

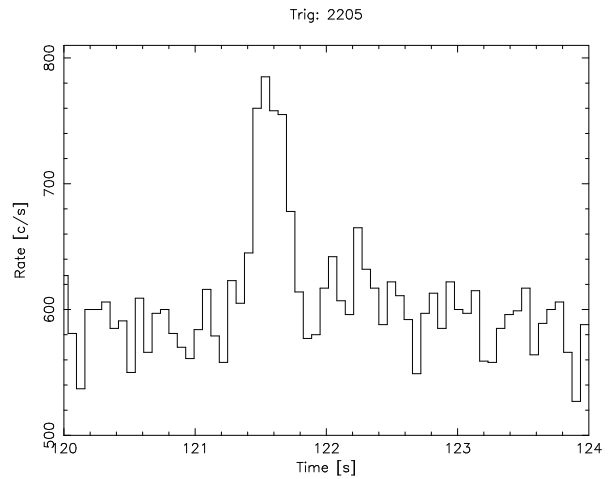

Figure 8: $64 \mathrm{~ms}$ light curve of the BATSE triger 2205. 